\title{
Clustering of random scale-free networks
}

\author{
Pol Colomer-de-Simon and Marián Boguñá \\ Departament de Física Fonamental, Universitat de Barcelona, Martí i Franquès 1, 08028 Barcelona, Spain
}

(Received 17 May 2012; published 30 August 2012)

\begin{abstract}
We derive the finite-size dependence of the clustering coefficient of scale-free random graphs generated by the configuration model with degree distribution exponent $2<\gamma<3$. Degree heterogeneity increases the presence of triangles in the network up to levels that compare to those found in many real networks even for extremely large nets. We also find that for values of $\gamma \approx 2$, clustering is virtually size independent and, at the same time, becomes a de facto non-self-averaging topological property. This implies that a single-instance network is not representative of the ensemble even for very large network sizes.
\end{abstract}

DOI: 10.1103/PhysRevE.86.026120

PACS number(s): 89.75.Fb, 05.70.Ln, 87.23.Ge

\section{INTRODUCTION}

Null models are critical to gauge the effect that randomness may have on the properties of systems in the presence of noise. It is therefore important to have the maximum understanding of the null model at hand, something not always easy to achieve. This is the case of the most used null model of random graphs, the configuration model (CM) [1-5].

Given a real network, the configuration model preserves the degree distribution of the real network, $P(k)$, whereas connections among nodes are realized in the most random way, always preserving the degree sequence - either the real one or one drawn from the distribution $P(k)$-and avoiding multiple and self-connections. In principle, the CM generates graphs without any type of correlation among nodes. For this reason, it is widely used in network theory to determine whether the observed topological properties of the real network might be considered as the product of some nontrivial principle shaping the evolution of the system.

This program is severely hindered when the network contains nodes with degrees above the structural cutoff $k_{s}=$ $\sqrt{\langle k\rangle N}$ [6], where $\langle k\rangle$ is the average degree and $N$ the size of the network. This is the case of scale-free networks with $P(k) \sim k^{-\gamma}, \gamma<3$, and a natural cutoff $k_{c} \sim N^{1 /(\gamma-1)}$ most often found in real complex networks [7]. This apparently simple null model develops all sort of anomalous behaviors in this case, e.g., the appearance of strong nontrivial degree correlations among nodes [6,8-10], difficulties in the sampling of the configuration space [11], or the presence of phase transitions between graphical and nongraphical phases [12], to name just a few.

Clustering - or the presence of triangles in the networkis yet another example of anomalous behavior associated with the CM. The importance of clustering as a topological property is related to the fact that nearly all known real complex networks have a very large number of triangles, whereas the CM has a vanishingly small number in the thermodynamic limit. Of course, the absence of triangles is convenient from a theoretical point of view as it allows us to use generating function techniques to solve many interesting problems [7]. However, given the empirical observations, it seems to be a quite unrealistic assumption. This has led to the common understanding that clustering observed in real networks cannot be explained by the CM and, thus, is the product of some underlying principle. While we fully agree with this statement, in this paper, we show that it must be taken with care. Indeed, depending on the heterogeneity of $P(k)$, the $\mathrm{CM}$ can generate, on average, nearly size-independent levels of clustering. In addition, in such cases, sample-to-sample fluctuations do not vanish when $N \rightarrow \infty$, meaning that the same degree sequence may generate either very high or very low levels of clustering, independently of the network size.

Clustering can be quantified using different metrics [13]. Here, we use the average clustering coefficient $C$, defined as the average (over nodes of degree $k \geqslant 2$ ) of the local clustering coefficient of single nodes $c_{i}=2 T_{i} / k_{i}\left(k_{i}-1\right)$, with $T_{i}$ the number of triangles attached to node $i$. In the absence of highdegree nodes, the clustering coefficient of a random graph generated by the CM is given by $[14,15]$

$$
C=\frac{\langle k(k-1)\rangle^{2}}{N\langle k\rangle^{3}},
$$

and, therefore, vanishes very fast at large system size. This is the reason why the treelike character of networks generated by the CM has always been taken for granted. However, Eq. (1) is clearly incorrect when the degree distribution is scale-free with a natural cutoff $k_{c} \sim N^{1 /(\gamma-1)}$, as it predicts a behavior $C \sim N^{(7-3 \gamma) /(\gamma-1)}$ that diverges for $\gamma<7 / 3$. Equation (1) fails in this case because its derivation does not account for the structural correlations among degrees of connected nodes. However, the same formula gives the correct scaling if, instead, a structural cutoff $k_{s} \sim N^{1 / 2}$ is imposed on the degree sequence. In this case, Eq. (1) predicts the correct scaling $C \sim N^{2-\gamma}$ [10]. It is then clear that the finite-size scaling of the clustering coefficient in random scale-free graphs must depend on both the size of the network $N$ and the particular scaling of the cutoff $k_{c}$ as a function of $N$. In this paper, we derive the correct scaling behavior of the clustering coefficient for scale-free random graphs with $2<\gamma<3$ and any cutoff value $k_{c}$.

\section{CLUSTERING IN MAXIMALLY RANDOM GRAPHS WITH EXPECTED DEGREE SEQUENCE}

The CM, as originally defined, defines a microcanonical ensemble, in the sense that the degree of every single node is given a priori and, once the degree sequence is fully 
known, the network is assembled in the most random way while preserving the degree sequence. (We refer the reader to Ref. [16] for a method to generate such graphs without any sampling bias.) However, in the case of scale-free networks, this approach resists any analytic treatment. Instead, here we adopt a different strategy and work with the canonical ensemble of the CM. In this ensemble, each node is given not its actual degree but its expected degree. This relaxes the topological conditions to close the network and opens the door to an analytic treatment. Specifically, the model is defined as follows:

(1) Each node is assigned a hidden variable $\kappa$ drawn from the probability density $\rho(\kappa) \propto \kappa^{-\gamma}$ with $1 \leqslant \kappa \leqslant \kappa_{c}$. The cutoff value $\kappa_{c}$ is, in principle, arbitrary. However, often $\kappa_{c}$ is the so-called natural cutoff, defined as the expected maximum value out of a sample of $N$ random deviates given from the probability density $\rho(\kappa)$. In the case of interest of a scale-free distribution, the natural cutoff scales as $\kappa_{c} \sim N^{1 /(\gamma-1)}$.

(2) Each pair of nodes is visited once and connected with probability

$$
r\left(\frac{\kappa \kappa^{\prime}}{\kappa_{s}^{2}}\right)=\frac{\kappa \kappa^{\prime}}{\kappa_{s}^{2}}\left(1+\frac{\kappa \kappa^{\prime}}{\kappa_{s}^{2}}\right)^{-1}
$$

where $\kappa$ and $\kappa^{\prime}$ are the hidden variables associated with each node, $\kappa_{s}=\sqrt{\frac{(\gamma-1) N\left(1-\kappa_{c}^{2-\gamma}\right)}{(\gamma-2) \bar{k}_{\min }}}$, and $\bar{k}_{\min }$ is the expected minimum degree of the network. The particular form chosen for the connection probability ensures that the entropy of the ensemble is maximal [17-19] (see also Appendix B).

It can be shown that the average degree of a node with hidden variable $\kappa$ is $\bar{k}(\kappa) \propto \kappa[9,20,21]$. Thus, we can think of $\kappa$ and $\rho(\kappa)$ as the degree and degree distribution, respectively [22].

Parameter $\kappa_{s}$ is a structural cutoff defining the onset of structural correlations, that is, nodes with expected degrees below $\kappa_{s}$ are connected with probability $r\left(\frac{\kappa \kappa^{\prime}}{\kappa_{s}^{2}}\right) \approx \frac{\kappa \kappa^{\prime}}{\kappa_{s}^{2}}$ and, therefore, are uncorrelated at the level of degrees. As a consequence, the global level of correlations present in the system is controlled by the cutoff $\kappa_{c}$. Whenever $\kappa_{c}<\kappa_{s}$ the resulting network is fully uncorrelated, whereas for $\kappa_{c} \geqslant \kappa_{s}$ correlations are necessary to close it. In this paper, we are interested in the range $\kappa_{s} \leqslant \kappa_{c} \leqslant N^{1 /(\gamma-1)}$.

Using the formalism developed in [20] (see also [24]), the local clustering coefficient of a node with hidden variable $\kappa$ can be written as

$$
c(\kappa)=\frac{\int_{\frac{1}{\kappa_{s}}}^{\frac{\kappa_{C}}{\kappa_{S}}} \int_{\frac{1}{\kappa_{S}}}^{\frac{\kappa_{C}}{\kappa_{s}}} \frac{1}{(x y)^{\gamma}} r\left(\frac{\kappa x}{\kappa_{s}}\right) r(x y) r\left(\frac{\kappa y}{\kappa_{s}}\right) d x d y}{\left[\int_{\frac{1}{\kappa_{s}}}^{\frac{\kappa_{C}}{\kappa_{s}}} x-\gamma^{2} r\left(\frac{\kappa x}{\kappa_{s}}\right) d x\right]^{2}} .
$$

The average clustering coefficient is computed from $c(\kappa)$ as $C=\int_{1}^{\kappa_{c}} \rho(\kappa) c(\kappa) d \kappa[25]$. However, $c(\kappa)$ is a bounded monotonically decreasing function and so its major contribution to $C$ comes from nodes with small degree, i.e., low $\kappa$ [10]. Therefore, to find the correct scaling behavior it suffices to evaluate $c(\kappa)$ in the domain $\kappa \ll \kappa_{s}$. In this case, the maximum value within the domain of integration $\left[1 / \kappa_{s}, \kappa_{c} / \kappa_{s}\right]$ of the arguments $\kappa x / \kappa_{s}$ and $\kappa y / \kappa_{s}$ in Eq. (3) is of order $O\left(\kappa_{c} / \kappa_{s}^{2}\right)$, which goes to zero in the thermodynamic limit. We can, thus, approximate $c(\kappa)$ as

$$
c(\kappa) \approx \frac{(\gamma-2)^{2}}{\kappa_{s}^{2(\gamma-2)}\left(1-\kappa_{c}^{2-\gamma}\right)^{2}} \int_{\frac{1}{\kappa_{s}}}^{\frac{\kappa_{c}}{\kappa_{s}}} \int_{\frac{1}{\kappa_{s}}}^{\frac{\kappa_{c}}{\kappa_{s}}} \frac{(x y)^{2-\gamma}}{1+x y} d x d y,
$$

which becomes independent of $\kappa$. After some manipulation, this expression becomes

$$
\begin{aligned}
c(\kappa) \approx & \frac{(\gamma-2)^{2}}{\kappa_{s}^{2(\gamma-2)}\left(1-\kappa_{c}^{2-\gamma}\right)^{2}}\left\{2 \psi(\gamma) \ln \left(\frac{\kappa_{c}}{\kappa_{s}}\right)+\theta(\gamma)\right. \\
& +\left(\frac{\kappa_{s}}{\kappa_{c}}\right)^{2(\gamma-2)} \Phi\left(-\left(\frac{\kappa_{s}}{\kappa_{c}}\right)^{2}, 2, \gamma-2\right) \\
& -2\left(\frac{\kappa_{c}}{\kappa_{s}^{2}}\right)^{3-\gamma} \Phi\left(-\frac{\kappa_{c}}{\kappa_{s}^{2}}, 2,3-\gamma\right) \\
& \left.+\frac{1}{\kappa_{s}^{6-2 \gamma}} \Phi\left(-\frac{1}{\kappa_{s}^{2}}, 2,3-\gamma\right)\right\},
\end{aligned}
$$

where

$$
\begin{aligned}
& \psi(\gamma)=\Phi(-1,1,3-\gamma)+\Phi(-1,1, \gamma-2), \\
& \theta(\gamma)=-\pi^{2} \cot \pi \gamma \csc \pi \gamma,
\end{aligned}
$$

and $\Phi(z, a, b)$ is the transcendent Lerch function [26]. This expression, although involved at first glance, is convenient because in the range $\kappa_{s} \leqslant \kappa_{c} \ll \kappa_{s}^{2}$ the arguments of the three transcendent Lerch functions in it go to $0^{-}$in the limit $\kappa_{s} \rightarrow$ $\infty$, in which case we know that $\Phi\left(-z^{2}, a, b\right) \sim b^{-a}$ for $z \rightarrow 0$. We then find the asymptotic behavior

$c(\kappa) \sim \frac{(\gamma-2)^{2}}{\kappa_{s}^{2(\gamma-2)}} \begin{cases}\theta(\gamma)+\Phi(-1,2, \gamma-2), & \kappa_{c}=\kappa_{s} \gg 1, \\ 2 \psi(\gamma) \ln \left(\frac{\kappa_{c}}{\kappa_{s}}\right), & \kappa_{c} \gg \kappa_{s} \gg 1 .\end{cases}$

The first line in this equation recovers the result found in [10] for scale-free networks without structural correlations$c(\kappa) \sim N^{2-\gamma}$ when $\kappa_{c}=\kappa_{s} \sim N^{1 / 2}$-whereas the second line predicts $c(\kappa) \sim N^{2-\gamma} \ln N$ when $\kappa_{c} \sim N^{1 /(\gamma-1)}$, which corrects the incorrect scaling behavior predicted by Eq. (1) in this case. Interestingly, this scaling is different from that found for a model of growing random scale-free graphs [27], again making evident the difference between equilibrium and nonequilibrium models of random graphs [28].

Figure 1 shows a comparison between numerical simulations, the numerical solution of Eq. (3) and the approximate solution given by Eq. (5), showing a very nice agreement. Interestingly, for $\gamma=2.1$, clustering remains nearly constant in the range of sizes $10^{3}-10^{5}$ and even increases slightly for small sizes. This is a consequence of the slow decay of the term $\kappa_{s}^{2(2-\gamma)}$ combined with the diverging logarithmic term in the numerator and functions $\psi(\gamma)$ and $\theta(\gamma)$, which diverges in the limit $\gamma \rightarrow 2$. In the inset of Fig. 1, we show the extrapolation of the clustering coefficient for sizes up to $10^{8}$ evaluated with Eq. (5). In the case of $\gamma=2.1$, this figure makes evident the extremely slow decay—nearly absent-with the system size. This implies that, in practice, clustering cannot be removed from the network even in very large networks when $\gamma \approx 2$. It is, thus, not clear whether the treelike approximation, customarily used to solve problems on random graphs, can be applied in this 


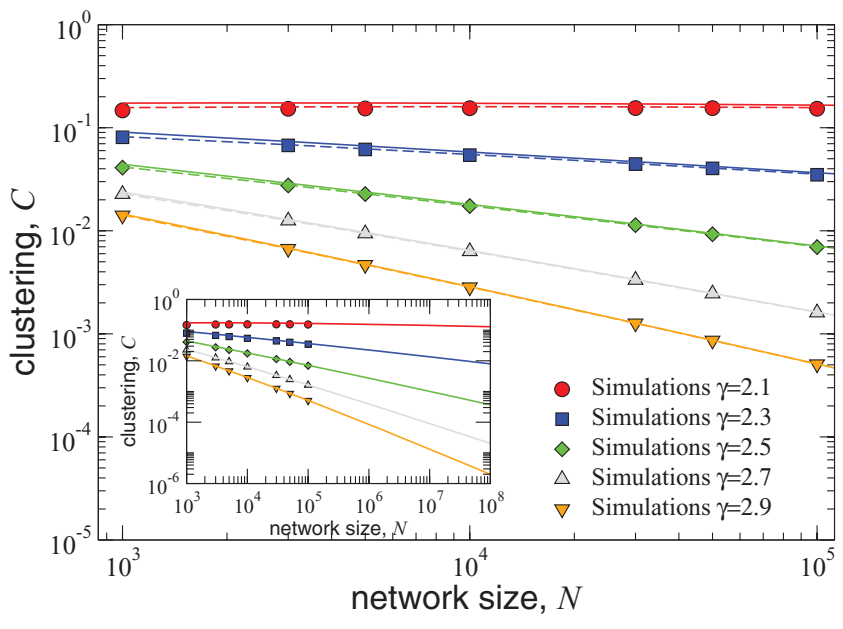

FIG. 1. (Color online) Clustering coefficient as measured in numerical simulations for different values of $\gamma$ and size $N$ with $\bar{k}_{\text {min }}=2$ and $\kappa_{c}=N^{1 /(\gamma-1)}$. Each point is an average over $10^{4}$ different network realizations. Dashed lines are the numerical solution of Eq. (3) and solid lines are the approximate solution given by Eq. (5). The inset shows an extrapolation up to size $N=10^{8}$ using Eq. (5).

case. In this situation, one should use alternative approaches, like the one developed in [21]. These results are particularly relevant due to the abundance of real networks with values of $\gamma \approx 2$. It is also interesting to study the behavior of clustering as a function of $\gamma$ for a fixed network size. Figure 2 shows this behavior for different values of $N$, confirming the results found in Fig. 1. Clustering increases as $\gamma$ decreases and converges to a constant and size independent value at $\gamma=2$.

Up to this point, we have been concerned only with the ensemble average of the clustering coefficient. However, the $\mathrm{CM}$ ensemble shows strong sample-to-sample fluctuations. Figure 3 shows the probability density function of the clustering coefficient obtained out of a sample of $10^{4}$ different networks generated by the canonical version of the CM. As

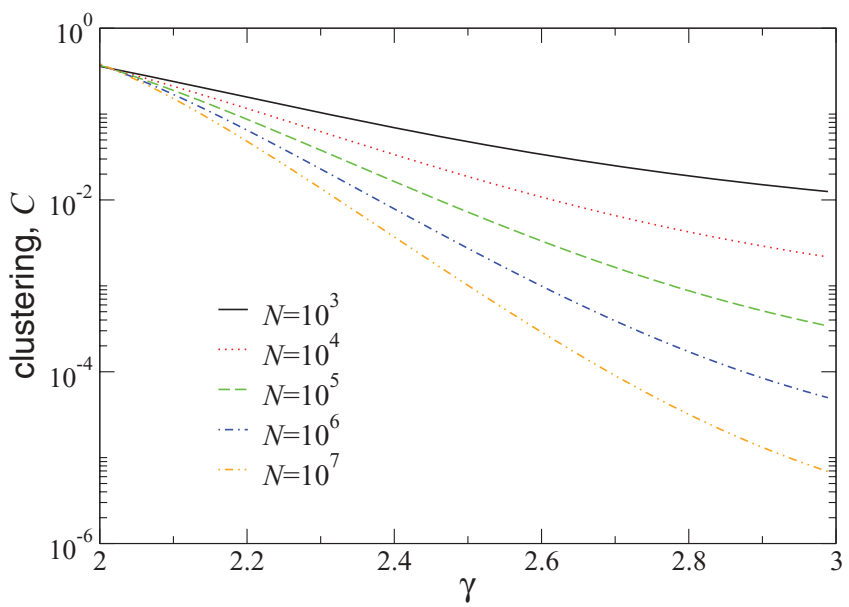

FIG. 2. (Color online) Clustering coefficient as a function of $\gamma$ for different network sizes. Curves are evaluated from Eq. (5) with $\bar{k}_{\min }=2$ and $\kappa_{c}=N^{1 /(\gamma-1)}$. (a)
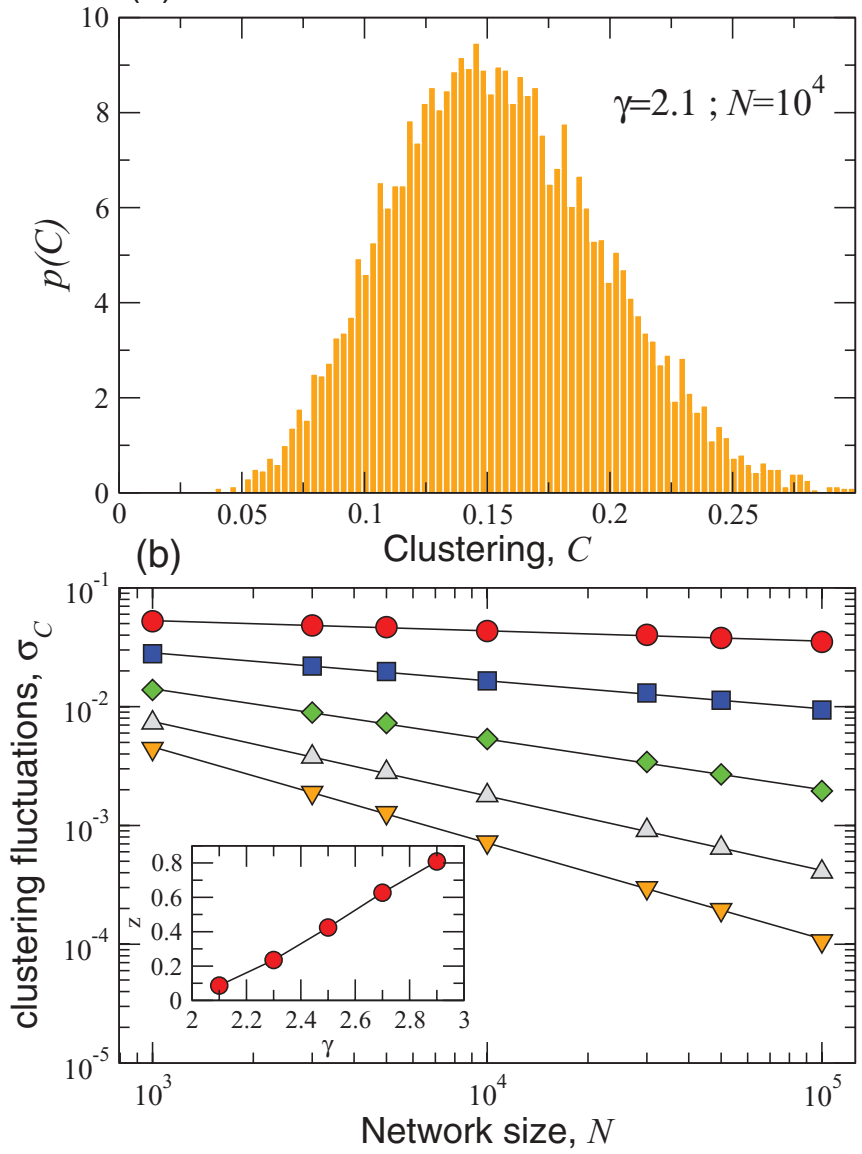

FIG. 3. (Color online) Sample-to-sample fluctuations. (a) shows the probability density function (PDF) of the clustering coefficient as obtained from $10^{4}$ network realizations for $\bar{k}_{\min }=2, \kappa_{c}=N^{1 /(\gamma-1)}$, $\gamma=2.1$, and $N=10^{4}$. (b) shows the standard deviation of this PDF for different values of $\gamma$ as a function of the network size. Solid lines are power law fits of the form $\sigma_{C} \sim N^{-z}$. The exponent $z$ is shown in the inset. Symbols in this figure means the same as in Fig. 1.

it can be observed, clustering may take values in the range $[0.05,0.25]$ quite easily. Figure 3 also shows the standard deviation $\sigma_{C}$ as a function of network size and for different values of $\gamma$. In all cases, fluctuations decay as a power law of the system size, $\sigma_{C} \sim N^{-z}$, with an exponent $z<1$. Interestingly, for $\gamma=2.1$, the exponent $z$ takes a very small value $(z \approx 0.1)$ that, when combined with the behavior of $C$ as a function of $N$, results in a nearly constant coefficient of variation. This implies that, in this range of values of $\gamma$, clustering is de facto a size-independent but non-selfaveraging property. That is, a single network instance is not a good representative of the ensemble even for very large network sizes.

\section{CONCLUSIONS}

The presence of triangles in real networks plays an important role in many processes taking place on top of them, e.g., percolation phenomena, epidemic spreading, synchronization, etc. It is, therefore, important to have full control over the 
most simple network ensembles that are used as null models to assess the presence of underlying principles shaping the topology of the system. In this paper, we have found the correct scaling behavior of the clustering coefficient of the ensemble of scale-free random graphs with $2<\gamma<3$. Interestingly, for values of the exponent $\gamma \approx 2$, clustering remains nearly constant up to extremely large network sizes. However, in this case, clustering is not self-averaging. This means that when comparing real networks against the $\mathrm{CM}$, it is not enough to generate a single-instance network, as it may result in either a very low or high level of clustering even for very large network sizes. These results are particularly important as the exponent $\gamma \approx 2$ seems to be-for yet unknown reasons-the rule rather than the exception in real systems.

\section{ACKNOWLEDGMENTS}

We thank M. Ángeles Serrano for useful comments and suggestions. This work was supported by MICINN Project No. FIS2010-21781-C02-02, Generalitat de Catalunya Grant No. 2009SGR838, and an ICREA Academia prize, funded by the Generalitat de Catalunya.

\section{APPENDIX A: DERIVATION OF EQ. (5)}

To derive Eq. (5) we first notice the following identity:

$$
\int_{0}^{a} d x \int_{0}^{b} d y \frac{(x y)^{2-\gamma}}{1+x y}=(a b)^{3-\gamma} \Phi(-a b, 2,3-\gamma)
$$

for $2<\gamma<3$ and $a, b>0$, which allows us to write Eq. (4) as

$$
\begin{aligned}
c(\kappa) \approx & \frac{(\gamma-2)^{2}}{\kappa_{s}^{2(\gamma-2)}\left(1-\kappa_{c}^{2-\gamma}\right)^{2}}\left\{\left(\frac{\kappa_{c}}{\kappa_{s}}\right)^{2(3-\gamma)} \Phi\left[-\left(\frac{\kappa_{c}}{\kappa_{s}}\right)^{2}, 2,3-\gamma\right]\right. \\
& -2\left(\frac{\kappa_{c}}{\kappa_{s}^{2}}\right)^{3-\gamma} \Phi\left(-\frac{\kappa_{c}}{\kappa_{s}^{2}}, 2,3-\gamma\right) \\
& \left.+\frac{1}{\kappa_{s}^{6-2 \gamma}} \Phi\left(-\frac{1}{\kappa_{s}^{2}}, 2,3-\gamma\right)\right\} .
\end{aligned}
$$

The first argument of the second and third transcendent Lerch functions in this equation goes to zero in the thermodynamic limit because $\kappa_{c} \ll \kappa_{s}^{2} \sim N$. However, the argument of the first Lerch function diverges unless $\kappa_{c} \sim \kappa_{s}$. Unfortunately, there is no known asymptotic behavior for the Lerch function for diverging arguments. To overcome this problem, we use the integral representation of function $\Phi\left(-z^{2}, 2,3-\gamma\right)$,

$$
\Phi\left(-z^{2}, 2,3-\gamma\right)=\int_{0}^{\infty} \frac{x e^{-(3-\gamma) x}}{1+z^{2} e^{-x}} d x .
$$

The domain of integration in Eq. (A3) can be separated into the subdomains $[0,2 \ln z]$ and $(2 \ln z, \infty)$ such that the function $\left(1+z^{2} e^{-x}\right)^{-1}$ can be expanded as a converging Taylor series in each subinterval. Once this trick is used, it is easy to derive the following identity:

$$
\begin{aligned}
\Phi\left(-z^{2}, 2,3-\gamma\right)= & z^{-2(3-\gamma)}[2 \psi(\gamma) \ln z+\theta(\gamma)] \\
& +\frac{1}{z^{2}} \Phi\left(-\frac{1}{z^{2}}, 2, \gamma-2\right) .
\end{aligned}
$$

Notice that this expression has a well-defined behavior when $z \gg 1$. Plugging this expression into Eq. (A2) results in Eq. (5).

\section{APPENDIX B: CANONICAL ENSEMBLES OF MAXIMALLY RANDOM GRAPHS}

The material of this appendix is original but rephrased from several other sources [17-19]. In general terms, an ensemble of random graphs is a collection of graphs generated with some random mechanism such that each particular graph instance $\mathbb{A} \equiv\left\{a_{i j}\right\}$ is generated with probability $P(\mathbb{A})$, where $\mathbb{A}$ is the adjacency matrix. The Shannon entropy of the ensemble can then be defined as

$$
S=-\sum_{\mathbb{A}} P(\mathbb{A}) \log P(\mathbb{A})
$$

Maximally random graph (canonical) ensembles are those maximizing the ensemble entropy while fixing the average of some network functions $\left\langle F_{n}(\mathbb{A})\right\rangle=\sum_{\mathbb{A}} P(\mathbb{A}) F_{n}(\mathbb{A})$. We call them canonical because only the averages of functions $F_{n}$ are fixed, as opposed to microcanonical ensembles, where the actual value of $F_{n}$ is fixed. The solution of this problem is given by

$$
P(\mathbb{A})=\frac{e^{\sum_{n} \alpha_{n} F_{n}(\mathbb{A})}}{Z},
$$

where $\alpha_{n}$ are Lagrange multipliers associated with $F_{n}$ and $Z$ is the partition function of the ensemble.

The case of interest in this paper corresponds to fixing the expected degree of each node, that is, quantities $k_{i}=\sum_{j} a_{i j}$, $i=1, \ldots, N$ are fixed on average. In this case, the probability of finding a particular graph $\mathbb{A}$ becomes

$$
P(\mathbb{A})=\frac{e^{\sum_{i} \alpha_{i} \sum_{j} a_{i j}}}{Z}=\prod_{i<j} \frac{e^{\left(\alpha_{i}+\alpha_{j}\right) a_{i j}}}{1+e^{\left(\alpha_{i}+\alpha_{j}\right)}} .
$$

Notice that this expression can be rewritten as

$$
P(\mathbb{A})=\prod_{i<j} p_{i j}^{a_{i j}}\left(1-p_{i j}\right)^{1-a_{i j}},
$$

where

$$
p_{i j}=\frac{e^{\left(\alpha_{i}+\alpha_{j}\right)}}{1+e^{\left(\alpha_{i}+\alpha_{j}\right)}}
$$

is the probability of the existence of a link between nodes $i$ and $j$. Notice that the factorization in Eq. (B4) implies that a network belonging to this ensemble can be generated by pairwise connection probabilities given by Eq. (B5). Finally, by redefining the Lagrange multipliers as $\kappa_{i}=\kappa_{s} e^{\alpha_{i}}$ we are led to Eq. (2).

We note that this canonical ensemble is not exactly the same as the configuration model, which fixes the actual degree distribution of the network. However, the canonical ensemble, while having the same statistical properties as the microcanonical one (as also happens between different ensembles in statistical mechanics when the thermodynamic limit is attained), can be studied analytically. In our opinion, this advantage makes this model even more interesting than the original configuration model. 
[1] B. Bollobas, Eur. J. Comb. 1, 311 (1980).

[2] A. Bekessy, P. Bekessy, and J. Komlos, Stud. Sci. Math. Hung. 7, 343 (1972).

[3] E. A. Bender and E. R. Canfield, J. Comb. Theory, Ser. A 24, 296 (1978).

[4] M. Molloy and B. Reed, Random Struct. Algorithms 6, 161 (1995).

[5] M. Molloy and B. Reed, Combinatorics, Probab. Comput. 7, 295 (1998).

[6] M. Boguñá, R. Pastor-Satorras, and A. Vespignani, Eur. Phys. J. B 38, 205 (2004).

[7] M. E. J. Newman, Networks: An Introduction (Oxford University Press, Oxford, 2010).

[8] Z. Burda and A. Krzywicki, Phys. Rev. E 67, 046118 (2003).

[9] J. Park and M. E. J. Newman, Phys. Rev. E 68, 026112 (2003).

[10] M. Catanzaro, M. Boguñá, and R. Pastor-Satorras, Phys. Rev. E 71, 027103 (2005).

[11] H. Klein-Hennig and A. K. Hartmann, Phys. Rev. E 85, 026101 (2012).

[12] C. I. Del Genio, T. Gross, and K. E. Bassler, Phys. Rev. Lett. 107, 178701 (2011).

[13] M. A. Serrano and M. Boguñá, Phys. Rev. E 74, 056114 (2006).

[14] M. E. J. Newman, in Handbook of Graphs and Networks, edited by S. Bornholdt and H. G. Schuster (Wiley-VCH, Berlin, 2003).

[15] G. Bianconi, G. Caldarelli, and A. Capocci, Phys. Rev. E 71, 066116 (2005).
[16] C. I. Del Genio, H. Kim, Z. Toroczkai, and K. E. Bassler, PLoS ONE 5, e10012 (2010).

[17] D. Garlaschelli and M. I. Loffredo, Phys. Rev. E 78, 015101 (2008).

[18] K. Anand and G. Bianconi, Phys. Rev. E 80, 045102 (2009).

[19] K. Anand, G. Bianconi, and S. Severini, Phys. Rev. E 83, 036109 (2011).

[20] M. Boguñá and R. Pastor-Satorras, Phys. Rev. E 68, 036112 (2003).

[21] M. A. Serrano, D. Krioukov, and M. Boguñá, Phys. Rev. Lett. 106, 048701 (2011).

[22] The exact form of the degree distribution was first given in [23], showing the asymptotic behavior $P(k) \sim k^{-\gamma}$ as expected.

[23] M. Á. Serrano, D. Krioukov, and M. Boguñá, Phys. Rev. Lett. 100, 078701 (2008).

[24] G. Bianconi and M. Marsili, Phys. Rev. E 73, 066127 (2006).

[25] Notice that, since $\kappa$ is only the expected degree, a node with, for instance, expected degree $\kappa=1$ may end up with an actual degree above 1 , and vice versa. This implies that all values of $\kappa$ contribute to the global clustering of the network and, thus, the domain of integration is $\left[1, \kappa_{c}\right]$.

[26] I. S. Gradstein and I. M. Ryzhik, Table of Integrals, Series, and Products, 6th ed. (Academic Press, San Diego, 2000).

[27] A. Barrat and R. Pastor-Satorras, Phys. Rev. E 71, 036127 (2005).

[28] S. N. Dorogovtsev and J. F. F. Mendes, Evolution of Networks: From Biological Nets to the Internet and WWW (Oxford University Press, Oxford, 2003). 